\title{
Evaluation of the Antioxidant Effects of Coffee and Its Components Using the Biomarkers Hydroxyoctadecadienoic Acid and Isoprostane
}

\author{
Yasukazu Yoshida*, Mieko Hayakawa and Etsuo Niki \\ Health Technology Research Center, National Institute of Advanced Industrial Science and Technology (AIST) (1-8-31 Midorigaoka Ikeda, \\ Osaka 563-8577, JAPAN)
}

\begin{abstract}
The association between coffee consumption and its antioxidant effects has not been elucidated in detail. In experimental animals, we used biomarkers to investigate the relationship between coffee consumption and its effects on oxidative stress. We propose a method in which both the free and ester forms of hydroperoxides and ketones as well as the hydroxides of linoleic acid are measured as total hydroxyoctadecadienoic acid (tHODE). Mice were divided into 6 groups: animals in 5 of these groups were fed a vitamin E-depleted diet [VE(-) group], whereas those in the $6^{\text {th }}$ (control) group were fed a diet containing 0.002 wt\% vitamin $E$ [VE(+) group]. Different $\mathrm{VE}(-)$ groups were also administered coffee or drinking water that contained a coffee component-chlorogenic acid, caffeic acid, or caffeine-for 1 month. It was clearly demonstrated that the liver levels of tHODE in the VE(-) groups increased compared to the $\mathrm{VE}(+)$ group but that coffee consumption reduced these elevated levels to that of the control. Interestingly, the plasma and liver levels of the HODE stereoisomer ratio $(Z, E / E, E)$, which is a measure of antioxidant capacity in vivo, were highest among the groups studied. These data, together with the values for antioxidant levels in vivo, indicate that the efficacy of antioxidants in vivo can be evaluated reasonably well based on the tHODE level and its stereoisomer ratio, and that the antioxidant capacity of coffee is superior to that of its individual components.
\end{abstract}

Key words: antioxidant, tHODE, isoprostanes, coffee

\section{INTRODUCTION}

Currently, it is generally accepted that lipid peroxidation is involved in the in vivo oxidative damage and pathogenesis of several disorders and diseases that are induced by reactive oxygen and nitrogen species. Lipid peroxidation may directly damage biological molecules and membranes, and may also induce the generation of toxic and signaling molecules ${ }^{1,2)}$. Accordingly, the potential role of antioxidant nutrients has been investigated with respect to the prevention of cancer, cardiovascular disease, cataracts, age-related macular degeneration, and aging. Coffee, in particular, has been received attention as a potent dietary antioxidant ${ }^{3,4)}$. From this viewpoint, lipid peroxidation products have received considerable attention as biomarkers for the evaluation of antioxidants. We have recently developed a method for the in vivo measurement of lipid peroxidation wherein total hydroxyoctadecadienoic acid (tHODE) is determined by gas chromatography-mass spectrometry (GC-MS) analysis of physiological samples after reduction with sodium borohydride followed by saponification with potassium hydroxide ${ }^{5)}$. Using this method, hydroperoxides and ketones as well as the hydroxides of both the free and ester forms of linoleic acid are measured as tHODE. Further, both free and ester forms of 8-iso-prostaglandin $\mathrm{F}_{2 \alpha}$ (8-iso-PGF $\mathrm{P}_{2 \alpha}$ ), one of the major forms of F2-isoprostanes, can be assessed simultaneously with tHODE by using GCMS. Another advantage of using tHODE as a biomarker is

Abbreviations: BSTFA, N,O-bis(trimethylsilyl)trifluoroacetamide; tHODE, total hydroxyoctadecadienoic acid; $(Z, E / E, E)$ HODE ratio, molar ratio of HODE stereoisomer, (9- and $13-(Z, E)-$ $\mathrm{HODE}) /(9-$ and $13-(E, E)-\mathrm{HODE})$; HPODE, hydroperoxyoctadecadienoic acid; t8-iso-PGF ${ }_{2 \alpha}$, total 8-iso-prostaglandin $\mathrm{F}_{2 \alpha}$; PBS, phosphate-buffered saline; $\alpha \mathrm{T}, \alpha$-tocopherol; $\gamma \mathrm{T}, \boldsymbol{\gamma}$-tocopherol

\footnotetext{
*Correspondence to: Yasukazu Yoshida, Health Technology Research Center, National Institute of Advanced Industrial Science and Technology (AIST), 1-8-31 Midorigaoka Ikeda, Osaka 563-8577, JAPAN E-mail: yoshida-ya@aist.go.jp
} Accepted July 31, 2008 (received for review March 31, 2008)

Journal of Oleo Science ISSN 1345-8957 print / ISSN 1347-3352 online

http://www.jstage.jst.go.jp/browse/jos/ 
that, in addition to its higher concentration than F2-isoprostanes, its regional and stereoisomers can be measured separately ${ }^{5}$. The ratio of cis-trans to trans-trans HODE is a measure of the capacity of hydrogen atom donation at the site of oxidation. This measure, i.e., the $(Z, E / E, E)$ HODE ratio, is practically important for the assessment and evaluation of the in vivo antioxidant capacity of natural and synthetic compounds, foods, and beverages, as well as supplements and their components $\mathrm{s}^{6-10}$. In the present study, we used tHODE and its stereoisomer ratio to investigate the antioxidative effects of coffee administered in drinking water. Furthermore, we also investigated the coffee components-chlorogenic acid, caffeic acid, and caffeine-in order to clarify the detailed mechanism of the antioxidative effect of coffee.

\section{EXPERIMENTAL PROCEDURES}

\subsection{Chemicals and preparation of coffee}

8-iso-PGF ${ }_{2 \alpha}, 8$-iso-prostaglandin $\mathrm{F}_{2 \alpha}-\mathrm{d}_{4}$ (8-iso- $\left.\mathrm{PGF}_{2 \alpha}-\mathrm{d}_{4}\right)$, 13-hydroxy-9(Z),11(E)-octadecadienoic acid (13- $(Z, E)$ HODE), 13-hydroxy-9(E),11(E)-octadecadienoic acid (13$(E, E)$-HODE), 9-hydroxy-10(E),12(Z)-octadecadienoic acid (9-(E,Z)-HODE), 9-hydroxy-10(E),12(E)-octadecadienoic acid (9-(E,E)-HODE), and $9(S)$-hydroxy-10(E),12(Z)octadecadienoic-9,10,12,13- $\mathrm{d}_{4}$ acid $\left(9-\mathrm{HODE}-\mathrm{d}_{4}\right)$ were obtained from the Cayman Chemical Company (MI, USA). Other materials such as coffee and its components, chlorogenic acid, caffeic acid, and caffeine, were of the highest grade commercially available. Coffee $(35 \mathrm{~g})$ was extracted with $1 \mathrm{~L}$ water using a coffee maker and its composition was assessed by high-performance liquid chromatography (HPLC) as described below.

\subsection{Animals and protocols}

Male mice (specific pathogen-free, C57BL/6J, weighing 19-24 g, and aged 11 weeks) were purchased from Nippon Clea Co. (Tokyo, Japan). They were divided into 6 groups according to the type of diet: a control diet containing 0.002 wt $\% \alpha$-tocopherol ( $\alpha \mathrm{T}$, control); a vitamin E-free (Efree) diet (Funabashi Nojyo, Chiba, Japan); and an E-free diet plus coffee $(35 \mathrm{~g} / \mathrm{L})$, chlorogenic acid $(150 \mathrm{mg} / \mathrm{L})$, caffeic acid $(10 \mathrm{mg} / \mathrm{L})$, or caffeine $(400 \mathrm{mg} / \mathrm{L})$ (Fig. 1). The composition of the E-free diet is summarized in our previous report ${ }^{6}$. The mice were maintained under standardized conditions of light (7:00 AM-7:00 PM), temperature $\left(22^{\circ} \mathrm{C}\right)$, and humidity (70\%). After 1 month, the animals were sacrificed under diethyl ether anesthesia. The experimental protocols were approved by the Institutional Animal Care and Use Committee (IACUC) of the National Institute of Advanced Industrial Science and Technology.

\subsection{HPLC analysis}

Plasma lipophilic antioxidants were extracted with chloroform-methanol $(2: 1, \mathrm{v} / \mathrm{v})$. Mouse liver and brain were homogenized in saline (liver:saline $=1: 3, \mathrm{w} / \mathrm{w}$ ) using a Polytron PT3100 tissue homogenizer (Kinematica AG, Lucerne, Switzerland). Chloroform-methanol (100 $\mu \mathrm{L}, 2: 1$, $\mathrm{v} / \mathrm{v}$ ) was added to the homogenized suspension ( $50 \mu \mathrm{L}, 25$ $\mathrm{w} / \mathrm{w} \%)$ and the lipids and vitamin $\mathrm{E}$ were extracted by centrifugation $\left(20400 \times g, 4^{\circ} \mathrm{C}, 10 \mathrm{~min}\right)$ after mixing vigorously using a vortex mixer. Subsequently, $\alpha \mathrm{T}$ and $\gamma \mathrm{T}$ were measured using an HPLC system equipped with an amperometric electrochemical detector (NANOSPACE SI-1; Shiseido Co., Ltd., Tokyo, Japan) set at $800 \mathrm{mV}$, combined with an octadecyl-bonded silica (ODS) column (LC18, $5 \mu \mathrm{m}, 250 \times$ 4.6 mm; Sigma-Aldrich Japan Com., Tokyo, Japan); methanol-tert-butyl alcohol (95:5, v/v) containing $50 \mathrm{mM}$

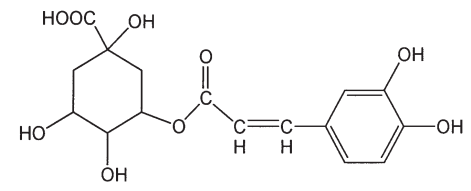

Chlorogenic acid

$(165.2 \mu \mathrm{g} / \mathrm{ml}, 559.5 \mu \mathrm{M})$

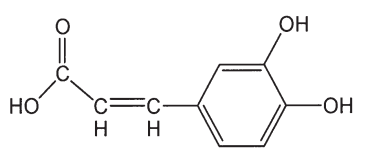

Caffeic acid

$(4.8 \mu \mathrm{g} / \mathrm{ml}, 32.0 \mu \mathrm{M})$<smiles>CN1c2ncn(C)c2C(O)N(C)C1O</smiles>

Caffein

$(502.4 \mu \mathrm{g} / \mathrm{ml}, 3104.6 \mu \mathrm{M})$

*Parenthesis shows the concentration in the coffee used in this study.

Fig. 1 The Structures of the Major Components and Their Concentrations in the Coffee Used in This Study.

Mice were divided into 6 groups according diet type: a control diet containing $0.002 \mathrm{wt} \% \alpha \mathrm{T}$ (control); a vitamin E-free (E-free) diet; and E-free diets plus drinking coffee (extracted as $35 \mathrm{~g} / \mathrm{L}$ ), chlorogenic acid $(150 \mathrm{mg} / \mathrm{L}$ ), caffeic acid $(10 \mathrm{mg} / \mathrm{L})$, or caffeine $(400 \mathrm{mg} / \mathrm{L})$ for 1 month. 
sodium perchlorate was used as the eluent at a flow rate of $1 \mathrm{~mL} / \mathrm{min}$. The levels of vitamin $\mathrm{C}$ in the plasma and homogenates of liver and brain were measured using an HPLC system equipped with a UV detector (SPD-10AV, 263 nm; Shimadzu Corp., Kyoto, Japan). An $\mathrm{NH}_{2}$ column (Wakosil $5 \mathrm{NH}_{2}, 5 \mu \mathrm{m}, 250 \times 4.6 \mathrm{~mm}$; Wako Pure Chemical Ind., Ltd., Tokyo, Japan) was used with $40 \mathrm{mM}$ PBSmethanol $(1: 9, \mathrm{v} / \mathrm{v})$ as the eluent delivered at a rate of 1 $\mathrm{mL} / \mathrm{min}$. Vitamin $\mathrm{C}$ was extracted from the samples with methanol (sample:methanol $=1: 4, \mathrm{v} / \mathrm{v}$ ) using the same method described above; an aliquot of the upper layer was immediately injected into the HPLC column. Ubiquinols and ubiquinones were also measured simultaneously according to a previously reported method ${ }^{13)}$. Briefly, they were measured using an HPLC system equipped with a NANOSPACE SI-1 detector set at $700 \mathrm{mV}$. The samples were passed through a reverse-phase column (LC8, $5 \mu \mathrm{m}$, $250 \times 4.6 \mathrm{~mm}$; Sigma-Aldrich Japan Co.) followed by a reducing column (RC-10, $30 \times 4 \mathrm{~mm}$; Shiseido Co., Ltd.); methanol-tert-butyl alcohol (85:15, v/v) containing $50 \mathrm{mM}$ sodium perchlorate was used as the eluent at a flow rate of $1 \mathrm{~mL} / \mathrm{min}$. Ubiquinols and ubiquinones were extracted from the plasma and homogenates of liver and brain with methanol-hexane (sample:methanol:hexane $=1: 5: 10$, $\mathrm{v} / \mathrm{v} / \mathrm{v}$ ); an aliquot of the upper layer was immediately injected into the HPLC column. The components of coffee were assessed as follows. Perchloric acid solution (60\%, 0.2 $\mathrm{mL}$ ) was mixed with $1 \mathrm{~mL}$ of coffee using a vortex mixer and the resulting solution was centrifuged for $10 \mathrm{~min}$ at $20400 \times g$ and $4^{\circ} \mathrm{C}$. An aliquot of the upper layer was injected into an HPLC system [Waters 2690 Separations Module with a Photodiode Array Detector (Waters 996)] equipped with an ODS-2, 4.6 × $150 \mathrm{~nm}$ column (GL Science, Co., Ltd., Tokyo, Japan) and was eluted using a gradient eluent [10 $\mathrm{mM}$ phosphate buffer (A) and acetonitrile (B): A 5\% to 70\% in $25 \mathrm{~min}$ ]. The assessment of coffee and its major components is summarized in Fig. 1, together with the corresponding chemical structures.

\subsection{Analyses of tHODE and 8-iso- $\mathrm{PGF}_{2 \alpha}$ in the plasma, erythrocytes, liver, and brain}

The tHODE and t8-iso- $\mathrm{PGF}_{2 \alpha}$ were measured as follows using a previously reported method with slight modification $^{5}$. Animal blood was collected from the inferior vena cava using a heparinized syringe, and the erythrocytes and plasma were separated by centrifugation $(1580 \times \mathrm{g}, 10 \mathrm{~min})$. Immediately after collection, the plasma $(0.2 \mathrm{~mL})$ was used for the analysis. The liver was also collected and stored at $-80^{\circ} \mathrm{C}$ until analysis. Internal standards $\left[8-\right.$ iso $-\mathrm{PGF}_{2 \alpha}-\mathrm{d}_{4}(100$ $\mathrm{ng})$ and $\left.9-\mathrm{HODE}-\mathrm{d}_{4}(100 \mathrm{ng})\right]$ and $1 \mathrm{~mL}$ of methanol were added to the plasma $(200 \mu \mathrm{L})$, followed by reduction with an excessive amount of sodium borohydride at room temperature for 5 min under nitrogen. The liver and brain homogenates $(25 \mathrm{wt} \%, 300 \mu \mathrm{L})$ were diluted with saline
$(1700 \mu \mathrm{L})$. The internal standards and $1 \mathrm{~mL}$ of methanol were added to this solution followed by reduction as described above. Subsequently, the reduced sample was mixed with $1 \mathrm{M} \mathrm{KOH}$ in methanol $(1 \mathrm{~mL})$ under nitrogen and incubated in a shaker at $40^{\circ} \mathrm{C}$ for $30 \mathrm{~min}$ in the dark. The sample was centrifuged $\left(1580 \times \mathrm{g}, 4^{\circ} \mathrm{C}, 10 \mathrm{~min}\right)$ and the supernatant was diluted with a 4 -fold volume of water (adjusted to $\mathrm{pH} 3$ beforehand) and acidified (pH 3) using $2 \mathrm{~N}$ $\mathrm{HCl}$. The acidified sample was centrifuged $\left(1580 \times g, 4^{\circ} \mathrm{C}\right.$, $10 \mathrm{~min}$ ) and the supernatant was subjected to solid-phase extraction (5). The eluted solution was evaporated with nitrogen gas and $30 \mu \mathrm{L}$ of a silylating agent, i.e., $(N, O$ bis $)$ trimethylsilyl trifluoroacetamide (BSTFA), was added to the dried residue. The solution was vigorously mixed using a vortex mixer for $1 \mathrm{~min}$ and then incubated at $60^{\circ} \mathrm{C}$ for 60 min in order to obtain trimethylsilyl esters and ethers. This solution was diluted with $70 \mu \mathrm{L}$ of acetone and an aliquot of the sample was then injected into a gas chromatography system (GC 6890 N; Agilent Technologies Co., Ltd., Palo Alto, CA, USA) equipped with a quadrupole mass spectrometer (5973 Network; Agilent Technologies Co., Ltd.) and a fused-silica capillary column (HP-5MS, 5\% phenyl methyl siloxane, $30 \mathrm{~m} \times 0.25 \mathrm{~mm}$; Agilent Technologies Co., Ltd.). Helium was used as the carrier gas at a flow rate of $1.2 \mathrm{~mL} / \mathrm{min}$. Temperature was programmed to increase from 60 to $280^{\circ} \mathrm{C}$ at a rate of $10^{\circ} \mathrm{C} / \mathrm{min}$. The injector temperature was set at $250^{\circ} \mathrm{C}$ and the temperatures of the transfer lines to the mass detector and ion source were 250 and $230^{\circ} \mathrm{C}$, respectively. Electron energy was set at 70 $\mathrm{eV}$. The amounts of $\mathrm{t}$ - $-\mathrm{iso}-\mathrm{PGF}_{2 \alpha}$ and tHODE were determined using fragment ions of $\mathrm{m} / z 481$ and 440, respectively. The internal standards used for the quantification of 8iso- $\mathrm{PGF}_{2 \alpha}$ and HODE were 8-iso- $\mathrm{PGF}_{2 \alpha}-\mathrm{d}_{4}(\mathrm{~m} / z=485)$ and 9 -HODE- $\mathrm{d}_{4}(m / z=444)$, respectively.

\subsection{Statistical analysis}

The data were statistically analyzed using an analysis of variance (ANOVA) followed by Tukey's test for multiple comparisons. Data are expressed as mean values \pm standard deviation (SD).

\section{RESULTS AND DISCUSSION}

In a previous study using mice, we observed the significant influence of the lipophilic antioxidants on biomarkers in the blood and tissues compared with a vitamin E-depleted diet ${ }^{11}$. Accordingly, in the present study, we administered coffee and its components, chlorogenic acid, caffeic acid, and caffeine, as a drinking water to mice fed a vitamin E-depleted diet for a relatively long period (1 month). As shown in Fig. 2, there was no significant change in body weight among the 6 groups during the course of the study. After 1 month, blood and tissues were used for the analysis 


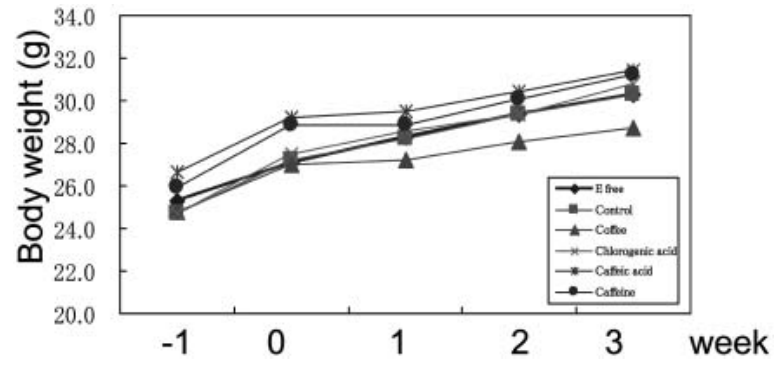

Fig. 2 Body Weights of Mice in the 6 Groups during the Study. The experimental conditions are similar to those described in Fig. 1.

of biomarkers.

3.1 Plasma levels of tHODE, t8-iso-PGF ${ }_{2 \alpha}$, and antioxidants

As shown in Fig. 3, the E-free diet increased the plasma levels of tHODE, whereas coffee, chlorogenic acid, caffeic acid, and caffeine decreased them. Thus, it was confirmed that the decrease in vitamin $\mathrm{E}$ increased lipid peroxidation in vivo and that each antioxidant tested was effective in reducing lipid peroxidation in vivo. The plasma levels of the antioxidants, namely, $\alpha \mathrm{T}, \gamma \mathrm{T}$, vitamin $\mathrm{C}$, ubiquinol, and ubiquinone, were also assessed. The results, summarized in Fig. 4, show that there were no significant differences among the different dietary groups with respect to the plasma levels of antioxidants measured in this study, except for $\alpha \mathrm{T}$. The stereoisomer ratio $(Z, E / E, E) \mathrm{HODE}$, which is a measure of the hydrogen donor capacity of antioxidants, decreased with the depletion of $\alpha \mathrm{T}$, but was recovered by consumption of coffee. The effect of $\alpha \mathrm{T}$ depletion and antioxidant supplementation did not, however, affect coenzyme $\mathrm{Q}$ levels.

\subsection{Liver levels of tHODE, t8-iso-PGF $2 \alpha$, and antioxidants}

The effects of the antioxidants were also observed in the liver. As shown in Fig. 5, the liver levels of tHODE and t8iso- $\mathrm{PGF}_{2 \alpha}$ exhibited a significant increase in response to a vitamin E-depleted diet, whereas the antioxidants lowered, although not significantly, these lipid peroxidation products to the control level. The liver levels of the antioxidants $\alpha \mathrm{T}, \gamma \mathrm{T}$, vitamin $\mathrm{C}$, ubiquinol, and ubiquinone were also assessed and the data are summarized in Fig. 6. There was no significant difference among the dietary groups with regard to the liver levels of ubiquinol-9 or ubiquinone9. Surprisingly, the antioxidant groups decreased the ascorbic acid levels in the liver. However, the stereoisomer ratio of HODE was increased by coffee consumption. Apparently, coffee and its components influenced the synthesis of ascorbic acid in vivo, but further study is needed for the elucidation of detailed mechanism.

The reactivity of radical-scavenging antioxidants in
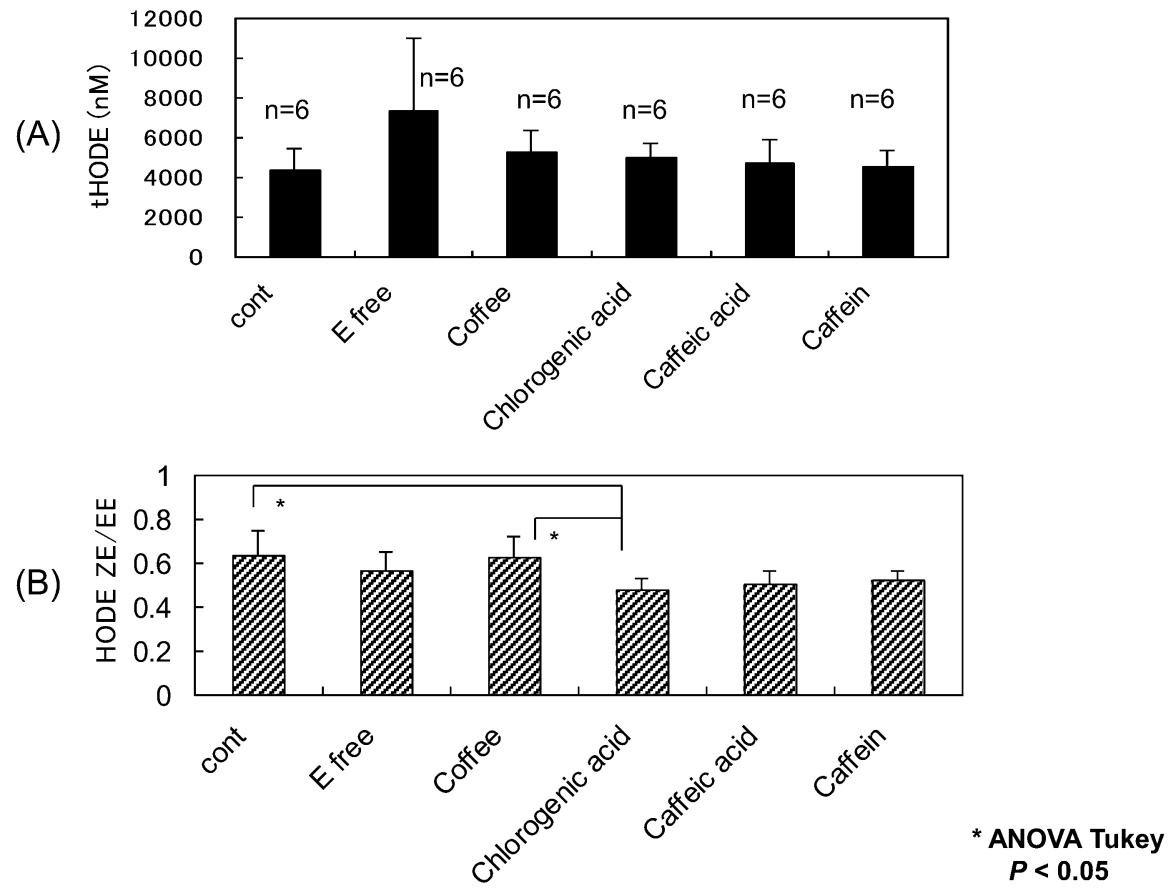

Fig. 3 Levels of tHODE (A) and the Stereoisomer Ratio of $\operatorname{HODE}(Z, E / E, E)(\mathrm{B})$ in the Plasma of Mice after 1 Month of the Test.

Experimental conditions are similar to those described in Fig. 1. 
(A)
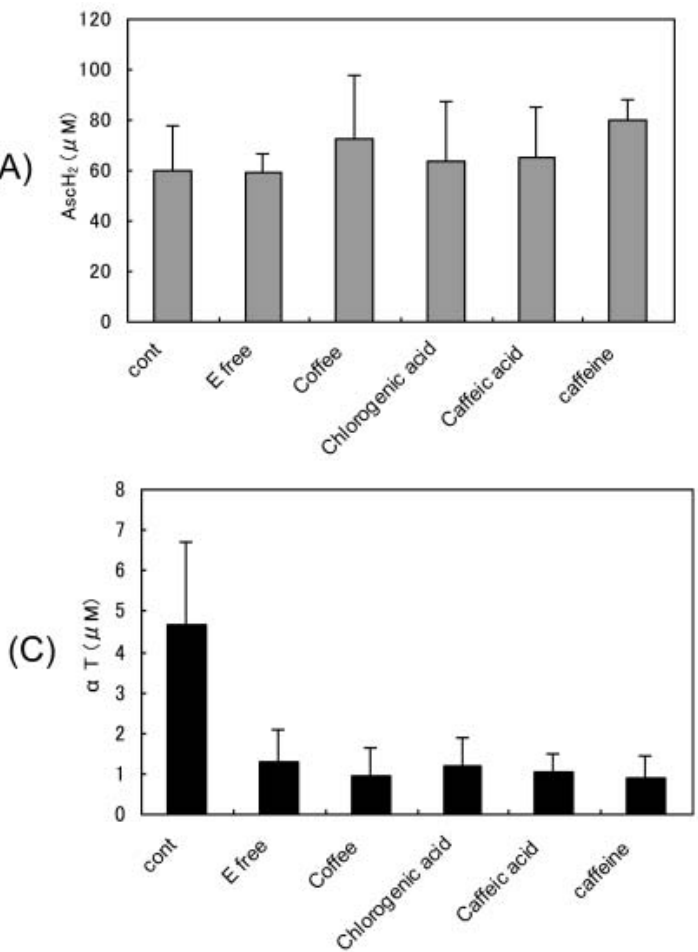

(B)

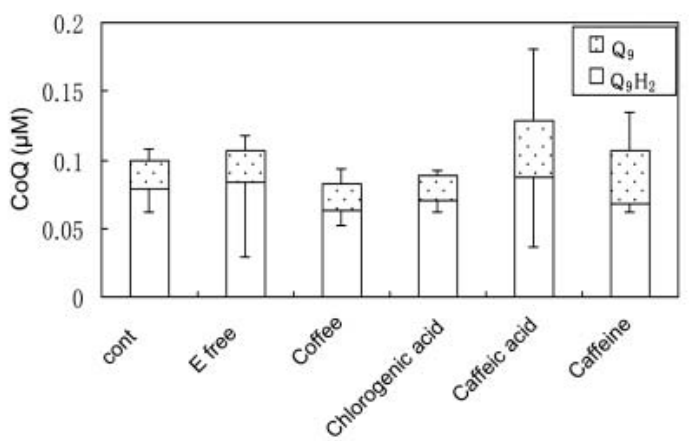

Fig. 4 Levels of Antioxidants, Vitamin C (A), Coenzyme $\mathrm{Q}_{9}$ (B), and $\alpha$-Tocopherol (C), in the Plasma.

Experimental conditions are similar to those described in Fig. 1. $\mathrm{Q}_{9} \mathrm{H}_{2}$, reduced form of coenzyme $\mathrm{Q}_{9}$; $\mathrm{Q}_{9}$, oxidized form of coenzyme $\mathrm{Q}_{9}$.

(A)

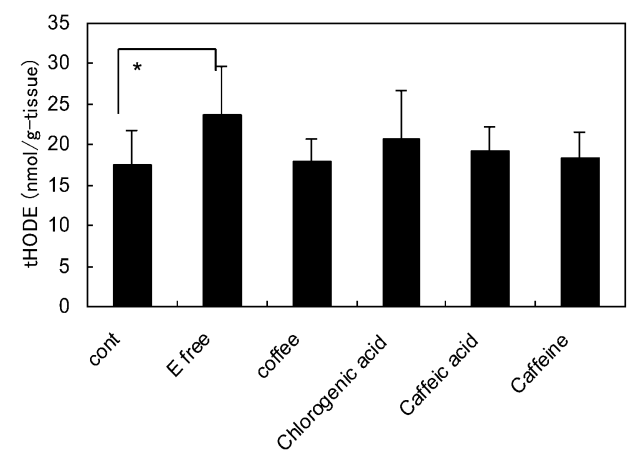

(C)

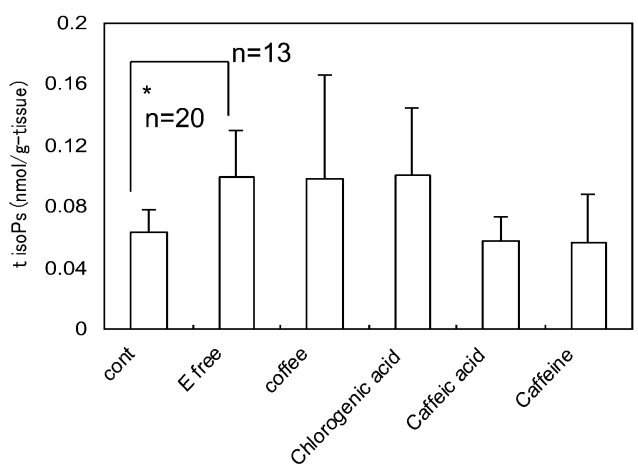

(B)

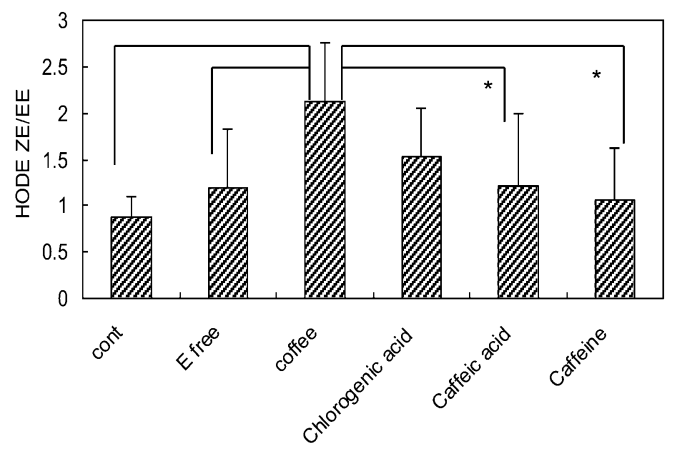

* ANOVA Tukey

Fig. 5 Levels of tHODE (A), Stereoisomer Ratio of $\operatorname{HODE}(Z, E / E, E)(\mathrm{B})$, and t8-iso-PGF $2 \alpha(C)$ in the Liver of Mice after 1 Month of the Test.

Experimental conditions are similar to those described in Fig. 1. 
(A)

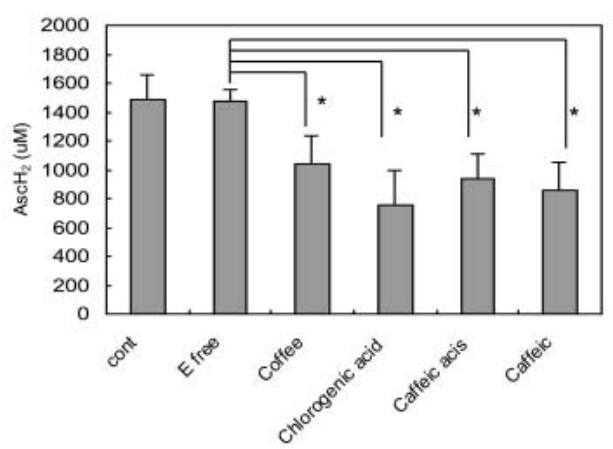

(C)

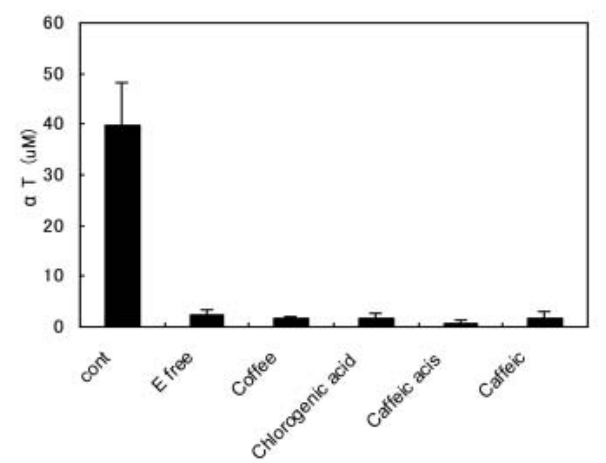

(B)

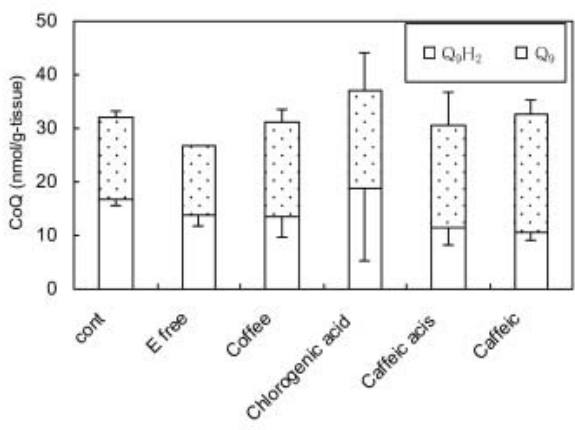

* ANOVA Tukey $P<0.05$

Fig. 6 Levels of Antioxidants, Vitamin C (A), Coenzyme $\mathrm{Q}_{9}(\mathrm{~B})$, and $\alpha$-Tocopherol (C), in the Liver.

Experimental conditions are similar to those described in Fig. 1. $\mathrm{Q}_{9} \mathrm{H}_{2}$, reduced form of coenzyme $\mathrm{Q}_{9}$; $\mathrm{Q}_{9}$, oxidized form of coenzyme $\mathrm{Q}_{9}$.

vitro may be determined quantitatively by several methods and the correlation with structure has been documented extensively for various antioxidants. However, the antioxidant efficacy in vivo is determined by several factors, such as concentration, localization, and mobility at the microenvironment level; the fate of antioxidant-derived radicals; interaction with other antioxidants; and bioavailability, that is, uptake, retention, metabolism, and excretion, as well as the chemical reactivity toward radicals ${ }^{14,15)}$. Therefore, the evaluation of antioxidant efficacy in vivo is difficult. One potential approach is to measure the effects of antioxidant supplementation or removal on the oxidative status by using biomarkers. In this study, experimental animals were used as an antioxidant evaluation model. In such studies, a vitamin E-depleted diet accelerates lipid peroxidation in vivo, which was observed in this study and also in previously reported studies ${ }^{6,11}$. Thus, this model is suitable for the evaluation of antioxidant mixtures in vivo using biomarkers as measures of lipid peroxidation. Chlorogenic acid and caffeic acid, which contain a phenol group, did not exhibit significant effects as antioxidants. This is an unexpected result as the phenol group is a wellknown antioxidant, at least in vitro. On the other hand, coffee, which contains both chlorogenic acid and caffeic acid exhibited antioxidant properties in both plasma and liver.
At present, the reason why the mixture exhibited this effect is unknown; however, it is assumed that an interaction among components probably plays a pivotal role in this property. This effect will hopefully be clarified in the near future.

The present study demonstrates that the efficacy of antioxidants in vivo can be evaluated reasonably well based on the tHODE level and its stereoisomer ratio, and that the antioxidant capacity of coffee is superior to that of its individual components.

\section{ACKNOWLEDGEMENT}

We cordially thank All Japan Coffee Association for the grant. We also thank Mrs. Yoko Habuchi for her excellent technique.

\section{References}

1. Leonarduzzi, G..; Arkan, M.C.; Basaga, H.; Chiarpotto, E.; Sevanian, A.; Poli, G. Lipid oxidation products in cell signaling. Free Rad. Biol. Med. 28, 1370-1378 (2000). 
2. Tang, D.G.; La, E.; Kern, J.; Kehrer, J.P. Fatty acid oxidation and signaling in apoptosis. Biol. Chem. 383, 425-442 (2002).

3. Anderson, L.F.; Jacobs, D.R.; Carlsen, M.H.; Blomhoff, $R$. Consumption of coffee is associated with reduced risk of death attributed to inflammatory and cardiovascular diseases in the Iowa Women's Health Study. Am. J. Clin. Nutr. 83, 1039-1046 (2006).

4. Sakamoto, W.; Isomura, H.; Fujie, K.; Takahashi, K.; Nakao, K.; Izumi, H. Relationship of coffee consumption with risk factors of aherosclerosis in rats. Ann. Nutr. Metab. 49, 149-154 (2005).

5. Yoshida, Y.; Niki, E. Detection of lipid peroxidation in vivo: Total hydroxyoctadecadienoic acid and 7-hydroxycholesterol as oxidative stress marker. Free Rad. Res. 38, 787-794 (2004).

6. Yoshida, Y.; Hayakawa, M.; Habuchi, Y.; Niki E. Evaluation of the dietary effects of coenzyme $Q$ in vivo by the oxidative stress marker, hydroxyoctadecadienoic acid and its stereoisomer ratio. Biochim. Biophys. Acta 1760, 1558-1568 (2006).

7. Yoshida, Y.; Itoh, N.; Hayakawa, M.; Piga, R.; Cynshi, O.; Jishage, K.; Niki, E. Lipid peroxidation induced by carbon tetrachloride and its inhibition by antioxidant as evaluated by an oxidative stress marker, HODE. Toxicol. Appl. Pharmacol. 208, 87-97 (2005).

8. Yoshida, Y.; Hayakawa, M.; Niki, E. Hydroxyoctadecadienoic acid as free radical-induced oxidative stress marker in vivo. BioFactors 24, 7-15 (2005).
9. Yoshida, Y.; Itoh, N.; Hayakawa, M.; Habuchi, Y.; Inoue, R.; Chen, Z.H.; Cao, J.; Cynshi, O.; Niki, E. Lipid peroxidation in mice fed choline-deficient diet and its inhibition by antioxidants as evaluated by an oxidative stress marker, HODE. Nutr. 22, 303-311 (2006).

10. Yoshida, Y.; Hayakawa, M.; Itoh, N.; Habuchi, Y.; Inoue, R.; Chen, Z.H.; Cao, J.; Cynshi, O.; Jishage, K.; Niki, E. Antioxidant effects of 2,3-dihydro-5-hydroxy-4,6- ditert-butyl-2,2-dipentylbenzofuran and $\alpha$-tocopherol in hyperlipidemic mice as evaluated by hydroxyoctadecadienoic acid and 7-hydroxycholesterol. Biochem. Pharmacol. 74,1010-1019 (2007).

11. Yoshida, Y.; Hayakawa, M.; Habuchi, Y.; Itoh, N.; Niki E. Evaluation of lipophilic antioxidant efficacy in vivo by the biomarkers hydroxyoctadecadienoic acid and isoprostane. Lipids 42, 463-472 (2007).

12. Yoshida, Y.; Saito, Y.; Hayakawa, M.; Habuchi, Y.; Imai, Y.; Sawai, Y.; Niki, E. Levels of lipid peroxidation in human plasma and erythrocytes: Comparison between fatty acids and cholesterol. Lipids 42, 439-449 (2007).

13. Yamashita, S.; Yamamoto, Y. Simultaneous detection of ubiquinol and ubiquinone in human plasma as a marker of oxidative stress. Anal. Biochem. 250, 66-73 (1997).

14. Niki, E. Antioxidants and atherosclerosis. Biochem. Soc. Trans. 32, 156-159 (2004).

15. Niki, E.; Noguchi, N. Evaluation of antioxidant capacity. What capacity is being measured by which method? IUBMB Life 50, 323-329 (2000). 